\title{
NUMEN Project:
}

Exploring key aspects of neutrinoless double beta decay by nuclear reactions

\begin{tabular}{|c|c|c|}
\hline $\begin{array}{l}\text { The Standard Model of particle } \\
\text { physics may represent our } \\
\text { most advanced understanding } \\
\text { yet of the universe's } \\
\text { fundamental building blocks, } \\
\text { but many physicists believe it } \\
\text { is incomplete. One of the most } \\
\text { enticing prospects for updating } \\
\text { the model lies with 'neutrino-less } \\
\text { double beta decay' - a process } \\
\text { which has been theorised for } \\
\text { many decades, but has yet } \\
\text { to be observed. Professor } \\
\text { Francesco Cappuzzello at } \\
\text { University of Catania and Dr } \\
\text { Clementina Agodi at National } \\
\text { Institute for Nuclear Physics } \\
\text { (Laboratori Nazionali del Sud } \\
\text { are the lead researchers of the } \\
\text { NUMEN Project. Representing } \\
\text { the efforts of a global team of } \\
\text { physicists, they ultimately aim } \\
\text { to unveil the key nuclear aspects } \\
\text { of neutrino-less double beta } \\
\text { decay for the first time. }\end{array}$ & $\begin{array}{l}\text { eta decay is one of the most well- } \\
\text { understood processes in subatomic } \\
\text { physics. It occurs when an atomic } \\
\text { cleus is composed of particular } \\
\text { rangements of protons and neutrons } \\
\text { ich make it unstable, causing it to } \\
\text { ther release a beta particle (an electron) } \\
\text { mm one of its neutrons, turning it into a } \\
\text { oton, or an anti-beta particle (a positron) } \\
\text { mm one of itt protons, transforming } \\
\text { nto a neutron. In some very rare cases, } \\
\text { uble beta decay can occur, where two } \\
\text { utrons will simultaneously transform } \\
\text { o protons, or vice versa, releasing either } \\
\text { o positrons or two electrons. } \\
\text { our current understanding of particle } \\
\text { yysics, defined by a set of rules called } \\
\text { e Standard Model, there are several } \\
\text { ndamental values which need to } \\
\text { conserved in every process, one } \\
\text { which is the 'lepton number'. Leptons } \\
\text { e a family of fundamental particles } \\
\text { ich include positrons and electrons, } \\
\text { ong with chargeless and extremely } \\
\text { ht particles, named neutrinos. Since } \\
\text { ectrons are 'matter' and positrons, } \\
\text { timatter', the Standard Model dictates }\end{array}$ & $\begin{array}{l}\text { that during beta decay, they must be } \\
\text { released alongside either an anti-neutrino } \\
\text { or a neutrino, respectively. } \\
\text { However, another type of beta decay } \\
\text { has been predicted theoretically where } \\
\text { this is not necessarily the case. In } \\
\text { 'neutrino-less double beta' (OVßB) decay, } \\
\text { two beta particles are emitted, but no } \\
\text { neutrinos. On the surface, this process } \\
\text { would appear to violate lepton number } \\
\text { conservation, but for several decades, } \\
\text { physicists have had reason to believe it } \\
\text { may still be possible. Therefore, observing } \\
\text { the process experimentally would have } \\
\text { profound consequences for our current } \\
\text { understanding of fundamental physics. } \\
\text { BREAKING THE STANDARD MODEL } \\
\text { In } 1937 \text {, Italian physicist Ettore Majorana } \\
\text { made an intriguing proposal: that it } \\
\text { could be possible for some particles to } \\
\text { be their own antiparticles. We know for } \\
\text { a fact that this can't be the case for a } \\
\text { majority of fundamental particles, since } \\
\text { one particle cannot have both positive } \\
\text { and negative charges, for example. } \\
\text { However, based on our currently- }\end{array}$ \\
\hline
\end{tabular}
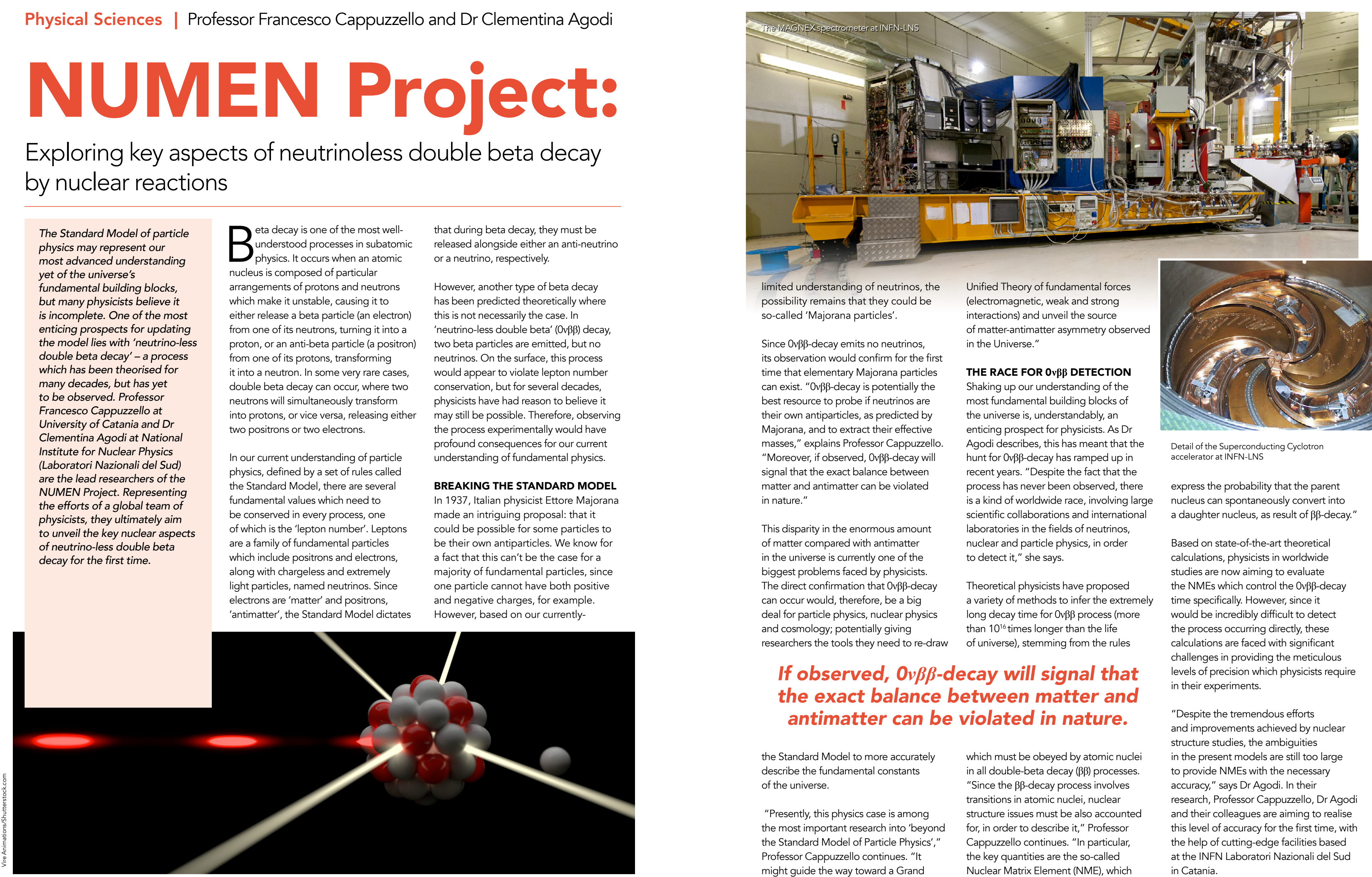

masses," explains Professor Cappuzzello. Agodi describes, this has meant that the

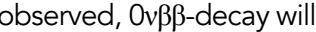
signal that the exact balance between matter and antimatter can be violated

This disparity in the enormous amount of matter compared with antimatter in the universe is currently one of the The direct confirmation that Ousicists. The dect contld the that Oupp-decay deal for partice physics, nucler physics and cosmology; potentially giving researchers the tools they need to hunt for $0 v \beta \beta$-decay has ramped up in recent years. "Despite the fact that the process has never been observed, there is a kind of worldwide race, involving large scientific collaborations and intemation laboratories in the fields of neutrinos, nuclear and particle physics, in order to detect it," she says.

Theoretical physicists have proposed a variety of methods to infer the extremely long decay time for Ov $\beta \beta$ process ( $\mathrm{m}$ of universe), stemming from the rules

If observed, Ovßß-decay will signal that the exact balance between matter and antimatter can be violated in nature.

the Standard Model to more accurately describe the fundamental constants

of the universe.

"Presently, this physics case is among the most important research into "beyon Professor Cappuzzello anticle Physic'" " might guide the way toward a Grand which must be obeyed by atomic nucle in all double-beta decay ( $\beta \beta$ ) processes. "Since the $\beta \beta$-decay process involves transitions in atomic nuclei, nuclear structure issues must be also accounted Cor, in order to describe it," Professor Cappuzzello continues. "In particular, Nuclear Matrix Element (NME), which
Detail of the Superconducting Cyclotron
accelerator at at NFN-LNS

express the probability that the parent nucleus can spontaneously convert into a daughter nucleus, as result of $\beta \beta$-decay."

Based on state-of-the-art theoretical calculations, physicists in worldwide he NMEs whic control the OpB the specifically However, since it would be incredibly difficult to itect the process occurring directly, these calculations are faced with significant challenges in providing the meticulous levels of precision which physicists require in their experiments.

"Despite the tremendous efforts and improvements achieved by nuclear structure studies, the ambiguities in the present models are still too large to provide NMEs with the necessary accuracy," says Dr Agodi. In their research, Professor Cappuzzello, Dr Agod and their colleagues are aiming to realise this level of accuracy for the first time, with the help of cutting-edge facilities based in Catania. 
OBSERVING A CLOSE ANALOGY physics where wo chas grocesses in simultaneously As Professor Cappuzzello explains, other 'Double Charge Exchange' (DCE) processes would be far easier to observe directly than OvBB-decay. "In this scenario, the experimental study of DCE reactions, which are processes promoting analogous nuclear transitions as $\beta \beta$-decay, could provide important information." he says. "The advantage is that DCEs can be studied under controlled

laboratory conditions."

Professor Cappuzzello and Dr Agodi's team take advantage of the fact that the processes undenlying Oupp-decay are comparable with those governing easierto-obsine DCE reactions. Therefore, the could be inferred by obsening DCEs occuring in one particul controllable environment: collisions between heavy fast-moving ions.

The team have attempted to do just this in previous studies. So far, however, their efforts have been hindered by the tiny probabilities by which the products of their heavy ion collisions were scattered $-a$ value named the collision 'cross-section'. With such imperceptible cross-sections, it has been incredibly hard for the physicists to know where to look for the collision products, where DCEs are occurring. But since these setbacks, new upgrades have been made to the NFN-

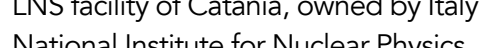
which could make their task far easier

With the new equipment, Professor Cappuzzello and Dr Agodi's team now hope to make cross-section measurements of heavy ion collisions far more easily. "Recently, we established an innovative experimental approach at the INFN-LNS laboratory in Catania, which allows us to largely overcome such experiment challenges, and consequently, to extract quantitative information on NIMEs fom DCE reactors, recounts Professor Cappuzzello.

\section{INTRODUCING:}

TUM PROJECT

In 2014, Professor Cappuzzello, Dr Agod

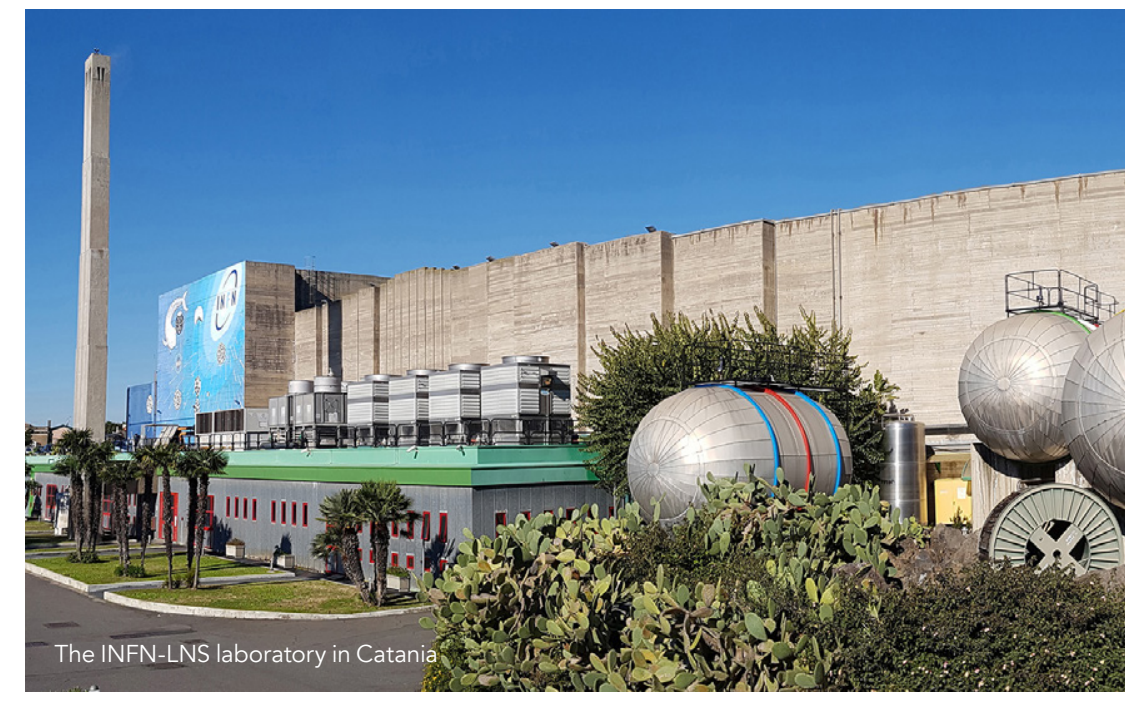

and their colleagues unveiled their plans for the NUclear Matrix Elements NUMEN) Project "The beta decay"

the NUMEN results, the researchers soon hope to embark on the most extensive arch for OußB NMEs ever undertaken. for this research in worldwide context, and will likely be in view of a major upgrade is to investigate the nuclear response explored by present and future studies of OvBB-decay," says Dr Agodi "Several aspects of the project require the development of innovative techniques, for both experiment set-up and theoretical interpretation of the collected data."

(s) is going to start in the coming months." says Professor Cappuzzello.

A PROMISING FUTURE

The NUMEN Project represents the coming together of minds encompassing At the heart of the new capabilities of the a diverse range of fields in physics. As NUMEN Project are two, state-of-the-art Professor Cappuzzello and Dr Agodi installations at the INFN LNS laboratory conclude, the researchers at NUMEN

NUMEN is a challenging project at the intersection of nuclear and neutrino physics, driven by an important physics case.

Catania. The first piece of equipment Cyclotron - a particle accelerator capable of accelerating heavy ions to high speeds, while ensuring precise, head-on collisions between ions. Secondly, the MAGNEX spectrometer will analyse the products of these collisions to incredibly high degrees of precision. "While K800 accelerates the heavy-ion beams with the required high resolution and low emittance,

MAGNEX detects the reaction products with large acceptance and high resolution in mass, energy and angle," Professor Cappuzzello explains.

With the help of these facilities, along with new cutting-edge theoretical calculation believe this is exactly what is needed to is a cha reng of nuclear and neutrino physics, driven by an important physics case and opening interesting scientific scenarios and potential technological fallout," they say.

In the coming years, the researchers involved with the project look set to be at the forefront of the global race to unvel OußB-decay experimentally, and provide the first clear evidence for the existence of Majorana particles. If they are successful a long-awaited shakeup to the Standard Model could finally be realised, allowing for an unprecedented oppotturity for

\section{Behind the Research 68

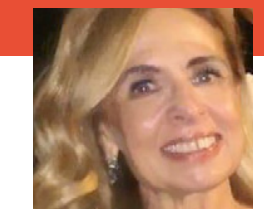

Professor Francesco Cappuzzello E: cappuzzello@|ns.infn.t.

T: +39095542384 (Office) +393281646400 (Mobile) W: $\frac{\text { www.dfa.unict.it/docenti//rancesco.cappuzzello }}{\text { W: } \text { https://www.Ins.infn.it// }}$

Dr Clementina Agodi E: agodi@Ins.infn.it

3283780365 (Mobile) W: $\frac{\text { www.researchgate.net/profile/Clementina_Ago }}{\text { W: https://www.Ins.infn.it// }}$.
W:

Research Objectives

The NUclear Matrix Elements for Neutrino-less 'double beta decay' (NUMEN) Project is an innovative project based at the INFN-Laboratori Nazionali del Sud. Using cutting edge technology, it aims to enhance our knowledge and understanding
of fundamental physics.

Detail

Professor Francesco Cappuzzello and Dr Clementina Agodi

Via S.Sofia n. 6295123

Catania, Italy

Clementina Agodi is an experimental reactions and structure of atomic nuclei. She holds a degree in Physics from Catania University and is cur researcher at INFN Laboratori Nazionali

del Sud. In $2007-2015$ she was member
of Nuclear Physics board of INFN.
Dr Agodi is Spokesperson of NUMEN
Project.
Francesco Cappuzzello is Associate
Professor of Experimental Nuclear
Physics of the Catania University
and Associate Researcher of the Istituto
Nazionale di Fisica Nucleare. He was
awarded his doctorate from the Catania
University in 1999, discussing a thesis

about the MAGNEX spectrometer

Funding

Nazionale di Fisica Nucleare (NFN), Università di Catania

\section{Collaborators}

- Dr Manuela Cavallaro INFN - LNS - The NUMEN Collaboration

\section{References}

Cappuzzello, F. Agodi, C. Cavallaro, M. Carbone, D. Tudisco, S. Presti, D.L. Oliveira, J.R.B. Finocchiaro, P. Colonna, M. Rifuggiato, D. Calabretta, L. et al. (2018). The NUMEN project The European Physical Journal A, 54(5) 72 .

Cappuzzello, F. Agodi, C. Carbone, D. Cavallaro, M. (2016) . Vergados, J.D. Ejiri, H. Simkovic, F. (2012). Theory in Physics 75(10) 106301. Dell'Oro, S. Marcocci, S. Viel, M. Vissani, F. (2016). Neutrinoless double beta decay: 2015 review. Advances

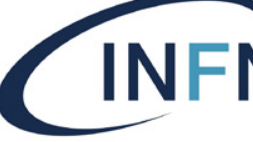

INFN

NUMEN
NUMEN

\section{Personal Response}

The NUMEN Project is a truly global collaboration. Can you give us a bit of the history behind how this

N in 2014 within the initiative, "WHAT NEXT?", in which INFN questioned itself on possible future avenues in the physics of fundamental

The project was based on a pioneering experiment, DOCET, F.Cappuzzello). NUMEN was considered worthy of the INFN challenge and was reviewed by a prestigious international evaluation committee, that strongly supported it. Initially, NUMEN attracted several colleagues, llowing us to con all the aspects of the challenge. Today, we are about 100
researchers from 35 Institutions in 15 countries.

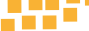

